\title{
Effects of cardio respiratory heated water-based training in an adult after heart transplantation
}

\begin{abstract}
Introduction: Heart transplant (HT) has been the technique of effective choice for the treatment of individuals with refractory heart failure. After HT, physical training becomes imperative in order to mitigate the muscular and cardiovascular impairment that is established. Hydrotherapy when performed in heated water has shown significant muscle and cardiovascular benefits.
\end{abstract}

Case report: A 38-year-old female volunteer, post-HT ( $<1$ year), underwent a heated water based exercise protocol of 10 weeks. Hemodynamic data, distance in the six minute walk test (6 MWT), velocity in the walking speed test (WST), respiratory and lower limb muscle strength and quality of life (QoL) were evaluated at baseline and post training.

Results: There was an increase in the muscular strength of the lower limbs and inspiratory muscles. In the $6 \mathrm{MWT}$, an increase in distance walked with a relative decrease of systolic blood pressure (SBP), diastolic blood pressure (DBP) and double product (DP) was observed. Furthermore, there was an increase in the left ventricle ejection fraction (LVEF). There was an increase in the walking speed observed in the $10 \mathrm{~m}$ WST. Regarding the QoL, an improvement in all domains of the SF-36 was observed.

Conclusion: The present study showed that an exercise protocol performed in warm water was safe and effective for improving hemodynamic variables, muscle strength, functional capacity and quality of life.

Keywords: heart transplant, hydrotherapy, blood pressure, heart rate, systolic volume and quality of life
Volume 3 Issue 2 - 2018

\author{
Freitas LSN, 'Araujo ML,' Ferreira JA,' \\ Borges VS,' Velloso EPP' \\ Physiotherapist in Physical Therapy Department, Universidade \\ de Itaúna (UI), Brazil
}

Correspondence: Freitas LSN, Physiotherapist in Physical Therapy Department, Universidade de Itaúna, Brazil, Email lusnfreitas@gmail.com

Received: January 10, 2018 | Published: April 20, 2018

\section{Introduction}

Heart transplantation (HT) has been the technique of effective choice for the treatment of individuals with refractory heart failure. ${ }^{1,2}$ In the last ten years, 2558 HTs were performed in Brazil, of which, 357 were performed between January and December 2016, with 178 of these carried out in the southeast region. ${ }^{2}$ Furthermore, it should be stated that heart transplanted cases still scarce in Brazil, when compared to worldwide. ${ }^{3}$

After receiving the transplanted heart, metabolic impairment is systemic, causing musculoskeletal damage, such as reduction of oxidative enzymatic activity and decreased capillarization. ${ }^{4}$ The occurrence of sarcopenia, atrophy, generalized muscle weakness, lower aerobic capacity and high blood pressure (HBP) is inherent, resulting from the hospitalization period and the physical inactivity and potentiated by the use of immunosuppressive drugs. ${ }^{4,5}$

To mitigate the pervasive harmful effects brought about by the HT guided exercises are prescribed. Exercises performed in water are one of the therapeutic modalities with exponential benefits. Aquatic physiotherapy has shown important muscle and cardiovascular benefits. ${ }^{6}$ Immersion in water may affect hemodynamic variables, as the external hydrostatic pressure increases the venous return, central blood volume and heart output (HO) with concomitant reduction of heart rate (HR) and peripheral vascular resistance (PVR). ${ }^{7}$ In addition to improvements in the renal and cardiac functions, systolic and diastolic blood pressure can be reduced at rest with physical training (PT) ${ }^{6-9}$

Considering the benefits of aquatic physiotherapy and the need for a well structured and differentiated rehabilitation for individuals submitted to HT, the aim of this study was to evaluate the cardio respiratory effects of an aquatic physiotherapy exercise program in a young adult after HT.

\section{Case characteristics}

This female volunteer was 38 years of age and had undergone a heart transplant in February 2016 due to dilated peripartum cardiomyopathy, in the Clinical Hospital $(\mathrm{CH})$ of Belo Horizonte. Two months after the HT, the subject was referred to the University of Itaúna Integrated Physiotherapy Clinics (CIFUI), located in Itaúna MG, to initiate Cardiovascular soil Rehabilitation for six months. After this period, due the particularly rare case and the benefits of water based exercises, this volunteer was invited to participate in this study.

\section{Materials and methods}

\section{Methods}

The study was approved by the Research Ethics Committee of the University of Itaúna, under authorization No. 1.843.378. The evaluations and the exercise program were carried out in the CIFUI. 
The initiation of the study occurred after authorization from the aforementioned clinic and the signing of the consent form by the volunteer.

\section{Strength test}

The microFet $2^{\circledR}$ manual dynamometer (Hoggan Health Industries, Salt Lake City, UT, USA) was used to evaluate the isometric strength of the plantar flexors, extensors, and knee flexors of the lower limbs (LLs). ${ }^{10}$ The manual dynamometer Takei ${ }^{\circledR} 5401$ (Yashiroda, Niigatashi, Japan) was used to evaluate manual grip strength. The isometric strength measurement was performed at baseline and after the end of the training. To begin the test of the knee extensors and flexors, the volunteer was positioned with hips and knees at $90^{\circ}$ flexion, the test was started with a range of motion (ROM) of $85^{\circ}$ of knee flexion-extension. In the plantar flexion test, the volunteer was placed in dorsal decubitus, with hip, knees and ankles in a neutral position and a dynamometer positioned between the anti-foot of the volunteer and the wall of the evaluation room. Finally, the manual pre-test was performed with the volunteer in orthostatism, with the upper limbs (ULs) in a neutral position, and dynamometer adjusted in the middle phalanges. As familiarization training, the volunteer performed two maximal contractions. Three maximal repetitions, maintained for five seconds were considered for the tests, with a rest interval of one minute between each effort. To stimulate the volunteer, standardized verbal feedback was given by the researcher responsible.

The dynamometers were properly calibrated and operationalized according to the manufacturer's instructions. All the measurement such as calibration, training or the researchers and placement were performed carefully.

\section{Functional capacity tests}

For the measurement of the functional capacity, the volunteer was first submitted to the 6-Minute Walk Test (6MWT), which was performed according to the American Thoracic Society ${ }^{11}$ recommendations, in a corridor of at least 50 meters and free of circulation of people. Next, the Walking Speed Test (WST) was carried out, in which the volunteer was instructed to walk 14 meters and the time, in seconds, was recorded, with the first two meters (acceleration phase) and the last two meters (deceleration phase) disregarded. ${ }^{12}$ Three measures of the time taken were collected.

\section{Respiratory muscle strength}

For the evaluation of respiratory muscle strength, the maximum inspiratory pressure (MIP) and the maximum expiratory pressure (MEP) were measured. For this, a $300 \mathrm{cmH}_{2} \mathrm{O}$ CriticalMed ${ }^{\circledR}$ manuvacuometer and nasal clip were used, having been sterilized. Measurements of MIP and MEP were measured according to Neder et al. ${ }^{13}$ Measurements were taken with a minimum interval of one minute between each.

\section{Quality of life}

Quality of life was assessed using the SF-36 multidimensional questionnaire, which consists of 36 items, encompassing eight domains: functional capacity, limitations in physical aspects, pain, general health status, vitality, social aspects, emotional aspects and mental health. It presents a final score for each item from 0 to 100 , where 0 corresponds to the worst general state of health and 100 the best state of health. ${ }^{14}$

\section{Borg scale}

The level of fatigue was assessed by the modified Borg scale, which consists of a vertical graded scale from 0 to 10 , with verbal expressions corresponding to a progressive increase in the level of perceived exertion, dyspnea or fatigue. ${ }^{15}$

\section{Ejection fraction (LVEF)}

The left ventricular ejection fraction (LVEF) was measured by means of a Doppler echocardiogram performed by the volunteer. The LVEF (\%) calculation was performed by the Teichholz method, which estimates the ejection fraction of the LV through the measurements of LV end-diastolic diameter (LVDD) and LV end-systolic diameter (LVSD). ${ }^{16}$ Two pre-HT scans (2011 and 2013), one post-HT (2016) and one exam after 10 weeks of PT in water (2017) were considered.

\section{Procedure}

After signing the consent form, an evaluation form was filled out containing demographic (name, age, gender) and clinical (previous history, medications in use and details about the health condition) data. Vital data such as blood pressure (BP), heart rate (HR), respiratory rate (RR), oxygen saturation $\left(\mathrm{SO}_{2}\right)$, and fatigue level measured by the Borg scale were collected, with the muscular strength and respiratory force tests being applied first. The volunteer then responded to the SF36 questionnaire, which was administered through an interview with a trained evaluator, with the participant in complete privacy. Once again, the vital data for the application of the 6MWT and WST were measured.

The evaluations were performed at two moments: baseline (preexercise) and after completing the 30 training sessions (end of the training)

The exercise protocol was performed in a 5.6 meter-long swimming pool heated to $33^{\circ} \mathrm{C}$, three times a week, for approximately 60 minutes per session. It was structured in three phases: Phase 1 adaptive, from the first to the third week, where a free walking warmup was performed without resistance, followed by walking training with resistance of $2 \mathrm{Kg}$ on both LLs, stationary bicycle and ending with relaxation where the volunteer was positioned horizontally with the use of floats. Phase 2 - intermediate, performed from the fourth to the sixth week, where there was an increase in resistance to $3 \mathrm{~kg}$, hydrodynamic dumbbells with resistance equivalent to $1 \mathrm{~kg}$ and step squatting exercise. Phase 3 - advanced, carried out from seventh to tenth week, with load increase to $4 \mathrm{~kg}$ on the ankle cuffs and $2 \mathrm{~kg}$ on the dumbbells, adding lateral walking with resistance ankle cuffs, stationary running, walking with abdominal resistance (aquajogger vest) and up and down stairs functional training. ${ }^{17,18}$ The intensity of the exercises was controlled through the perceived exertion scale between $4-6^{15}$ and saturation level $\left(\mathrm{SO}_{2}\right)$ above $90 \%$.

Immersion was made to the level of the xiphoid process. Throughout the training, the volunteer was monitored by two trained researchers. Vital data such as HR (heart rate monitor Oregon ${ }^{\circledR}$ - HR102), BP(stethoscope Littmann ${ }^{\circledR}$ and sphygmomanometer TENSO med $\left.{ }^{\circledR}\right)$ and saturation level (NONIN®onyx 9500) were measured before, during (every ten minutes) and after each session. The volunteer was allowed a rest period between activities, if necessary.

\section{Data analysis}

Descriptive statistics including mean (standard deviation), percentage and frequency were used to present the results. 


\section{Results}

The 38-year old volunteer, eight months after HT due to dilated cardiomyopathy, had a body mass of $83 \mathrm{~kg}$ and body mass index (BMI) of $28.42 \mathrm{~kg} / \mathrm{m}^{2}$. During the exercise program, the volunteer used calcium carbonate, sodium alendronate, antibacterials, mycophenolate mofetil, immunosuppressants, corticosteroids, antiallergics, ferrous sulfate and antihypertensives.

The muscle strength test, in Kgf, showed gains of more than $220 \%$ for knee flexor strength and above $100 \%$ for left knee extensors after compliance with the training protocol, with gains of over $12 \%$ in all muscle groups tested. Table 1 shows the percentage of strength gain in all muscle groups evaluated before and after the PT in water.

Table I Descriptive data of isometric muscle strength using portable hand dynamometer in Kgf (force production in kilograms). Considering the three repetitions and using the mean of the three measures, the Standard deviation and percentage of gain were used for the demonstration of the results

\begin{tabular}{llll}
\hline Muscle group & baseline & After training & \% gain \\
\hline Right knee Flex. & $3.40(0)$ & $12.10(0.6)$ & 255.90 \\
Left knee Flex. & $2.60(0.3)$ & $8.40(0.4)$ & 223.10 \\
Right knee Ex. & $10.70(1.1)$ & $14.96(2.3)$ & 39.81 \\
Left knee Ex. & $6.03(0.3)$ & $12.20(1.3)$ & 102.30 \\
Right plantar Flex. & $25.70(2.2)$ & $30.70(3.7)$ & 19.50 \\
Left plantar Flex. & $13.40(5.5)$ & $22.00(0.9)$ & 64.20 \\
Right palmar Grasp & $22.60(I .1)$ & $25.40(I .6)$ & 12.40 \\
Left palmar Grasp & $21.60(1.0)$ & $27.00(I .3)$ & 25.00 \\
\hline
\end{tabular}

Note values are expressed as mean (standard deviation)

In the 10 meter walking speed test a reduction of more than 8 seconds to complete the test was observed (baseline: $18.2 \mathrm{~s}$, postintervention: 10.3s) with no change in $\mathrm{HR}$ (mean of $86 \mathrm{bpm}$ and $\mathrm{SpO}_{2}$ 98\%).

Quality of life, evaluated through the SF-36 questionnaire, confirmed the beneficial effects of the physical gains (Table 2) (Table 3).

Table 2 Descriptive data on the quality of life presented according to the SF36, before and after the PT

\begin{tabular}{llll}
\hline Domain & Baseline & After training & \% gain \\
\hline Functional Capacity & 15.00 & 45.00 & $200.00 \%$ \\
Limitations in Physical & 0.00 & 75.00 & $75.00 \%$ \\
Aspects & 31.00 & 41.00 & $32.00 \%$ \\
Pain & 37.00 & 72.00 & $94.60 \%$ \\
General Health Status & 45.00 & 80.00 & $77.78 \%$ \\
Vitality & 25.00 & 50.00 & $100.00 \%$ \\
Social Aspects & 0.00 & 33.34 & $33.34 \%$ \\
Emotional Aspects & 44.00 & 64.00 & $45.45 \%$ \\
Mental Health & & & \\
\hline
\end{tabular}

In relation to the 6MWT, the distance covered at baseline was 240 meters, with this increasing to 309 meters after the PT. A large gain in exercise capacity was observed, as well as a relative decrease in Ratepressure (RP). The details are presented in (Table 4).

Left ventricular ejection fraction (LVEF) values are shown in Figure 1. The volunteer was submitted to four Doppler echocardiography examinations. The first two values (2011 and 2013) refer to the pretransplantation period, while the third value (2016) corresponds to post-transplantation moment ( 2 months after) and finally the last value (2017) is related to the post-training moment. There was an improvement of $14 \%$ in LVEF (\%) with $56 \%$ at baseline and $70 \%$ post-training.

Table 3 Descriptive data of maximal inspiratory and expiratory muscle strength, before and after the exercise protocol. The data from the six repetitions (mean, standard deviation and percentage of gain) were considered for the demonstration of the results

\begin{tabular}{llll}
\hline Variable & Baseline & After training & \% gain \\
\hline MIP & $76.7(I 2)$ & $120.0(12.7)$ & $56.45 \%$ \\
MEP & $80.0(22)$ & $88.3(\mid 1.7)$ & $10.37 \%$ \\
MIP\% & 98.0 & 141.6 & $44.50 \%$ \\
MEP\% & 108.2 & 108.2 & $0 \%$ \\
\hline
\end{tabular}

Note values are expressed as mean (standard deviation)

MIP, maximal inspiratory pressure; MEP: maximal expiratory pressure

Table 4 Results of the variables in the 6MWT at baseline and after 10 weeks of training.Variables were measured at rest and at the end of the 6MWT

\begin{tabular}{lll}
\hline Variables & Baseline & After I 0 weeks \\
\hline SBP $(\mathrm{mm} / \mathrm{hg})$ & Rest: I20 End: I30 & Rest: I I0 End: I 20 \\
$\mathrm{DBP}(\mathrm{mm} / \mathrm{hg})$ & Rest: 90 End: I I0 & Rest: 80 End: 80 \\
$\mathrm{HR}(\mathrm{mean}, \mathrm{bpm})$ & 82 & 98 \\
$\begin{array}{l}\text { Rate-pressure (bpm. } \\
\mathrm{mmHg})\end{array}$ & $\begin{array}{l}\text { Rest: } 9840 \text { End: } \\
\text { II I80 }\end{array}$ & $\begin{array}{l}\text { Rest: } 9460 \text { End: } \\
\text { I0320 }\end{array}$ \\
$\begin{array}{l}\text { SpO } \\
\text { Perception of Effort }\end{array}$ & 99 & 98 \\
$\begin{array}{l}\text { (Borg) } \\
\begin{array}{l}\text { Distance traveled } \\
\text { (meters) }\end{array}\end{array}$ & Rest: 0 End: 2 & Rest: 0 End: 2 \\
\hline
\end{tabular}

$H R$, heart rate; DBP, diastolic blood pressure; SBP, systolic blood pressure; $\mathrm{SpO}_{2}$, peripheral oxygen saturation; Rate-pressure, $\mathrm{BP}$, blood pressure

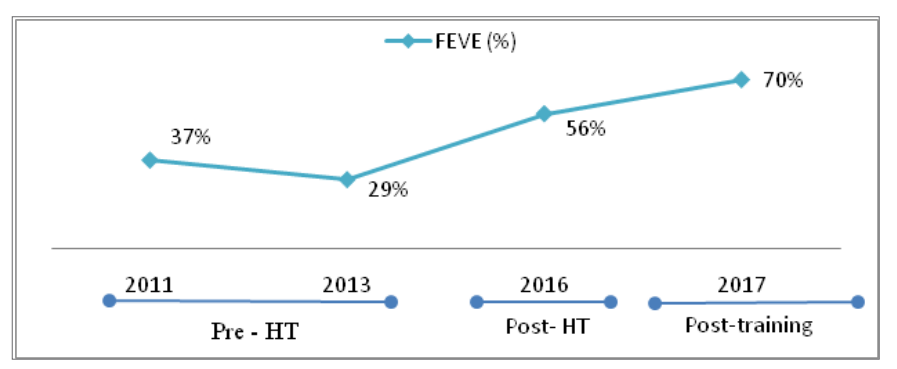

Figure I Percentage of LVEF pre-HT, post-HT and after 10 weeks of training. LVEF, left ventricular ejection fraction

\section{Discussion}

The results obtained in this case study suggest that a 10 -week training protocol in a heated pool was effective in increasing muscle strength, functional capacity, conditioning and walking speed. Regarding the hemodynamic variables, a relative reduction of SBP was observed from day 11. There was a reduction of the DP in the 6MWT and a significant improvement in the left ventricular ejection fraction (LVEF) of the myocardium in the post PT period. In addition to the physical gains, an improvement in the quality of life was noted, with gains of more than $30 \%$ in all domains of the SF-36 questionnaire. 


\section{Inspiratory muscular strength}

Patients undergoing cardiac surgery with extracorporeal circulation (ECC) usually present a reduction in respiratory capacity and respiratory muscle strength (RMS) after surgery. The effects of the surgery on muscle function, pain, and the presence of thoracic drains may explain this reduction and may result in impairment of respiratory function. ${ }^{19,20}$

Another relevant point is the previous history of dilated cardiomyopathy with congestive heart failure (CHF), which also contributes to the weakness of the inspiratory musculature. ${ }^{21,22}$ In this way, a condition of exercise intolerance and dyspnea are installed, even in relation to performing activities of daily living (ADL). ${ }^{22}$ The present study demonstrated that the PT resulted in an inspiratory muscle strength gain of $56.45 \%$, although the MIP values were within the normal range. The probable explanation for this fact would be previous training that the volunteer performed with another groundbased exercise protocol. ${ }^{10}$

Increased RMS may contribute to improved dyspnea and exercise tolerance, in order to delay diaphragmatic fatigue. ${ }^{23}$ In this context, other studies have demonstrated that there is a direct relationship between the increase in RMS and peripheral muscle strength. ${ }^{23,24}$ Thus, the present results suggest that the RMS improvement may also have contributed to improved peripheral muscle strength..$^{24,25}$

\section{Muscle strength of LLs}

It has been well described in the literature that post-HT patients exhibit a reduction in peripheral muscle strength (PMS) and increased systemic vascular resistance that can be attributed to the persistence of pre- and post-transplant abnormalities such as physical deconditioning, neuroendocrine abnormalities and the use of immunosuppressant medications. ${ }^{26}$

According to Pereira et al. ${ }^{22}$ The effects of a water-based exercise program on muscle strength and functionality, include the improvement of the ability of the muscles to produce torque. The combination of the use of resistance and the turbulence of the water created during the exercises, offer additional mechanical stimuli for the gain in strength. In this way, both the walking and the impediment of the speed of the movements in the water result in greater torques at the moment of propelling the body forward. ${ }^{27}$

In the present study, the symmetrical gain of more than $200 \%$ in the knee flexors reflects the exercise protocol that was based on walking training exercises with free weight association on the ankles, starting at $1 \mathrm{~kg}$ and increasing gradually to $4 \mathrm{~kg}$ ankle cuffs on both LLs. Thus, the water resistance imposed at both the moment of the balance phase and the support phase, as well as when the body is propelled forward during walking, led to a sustained contraction of the knee and hip flexors being required. In addition, at the time of the heel shock and medium support, there was a need for an important contraction of the flexors to prevent the body from retreating backwards due to the water turbulence. Thus, hypertrophy of the quadriceps was bilaterally achieved through this exercise protocol

These data are in agreement with Cuoco et al. ${ }^{27}$ who showed the greater effectiveness of water-based exercises in promoting muscle strength gain in older adults. ${ }^{28}$

Taken together, the results of the RMS improvement associated with PMS suggest an improvement in exercise tolerance that led to increased speed and distance walked in the 6MWT.

\section{Six-minute walk test}

At baseline, the distance traveled in the $6 \mathrm{MWT}$ was $240 \mathrm{~m}$, with mean velocity of $0.67 \mathrm{~m} / \mathrm{s}$. After the HT, there was an increase in the distance traveled to $309 \mathrm{~m}$, with a consequent increase in the mean speed to $0.85 \mathrm{~m} / \mathrm{s}$. This increase resulted in a lower cardiovascular risk for the patient, after the HT. ${ }^{29,30}$

In this context, Chen et al. ${ }^{28}$ observed that the shorter distances traveled in the 6MWT were associated with higher uric acid levels and a higher risk of death or hospitalizations for cardiovascular morbidity 1 and 3 years after the HT (29). In addition, they described a walking distance of $539 \pm 108 \mathrm{~m}$, about $89.9 \pm 18.2 \%$ of the predicted value for this test in post-HT patients (4.8 \pm 3.2$)$. There was a positive correlation between walking distance and $\mathrm{VO}_{2} \mathrm{HR}$ and peak SBP during the maximal cardiopulmonary test. Additionally, the authors described a strong correlation between distance and quality of life measured through the SF-36, mainly in the areas of general health perception and the physical component. ${ }^{29}$

On the other hand, Castel et al..$^{30}$ reported that a distance of less than $225 \mathrm{~m}$ walked in patients with moderate to severe heart failure would present a significant risk of mortality in the medium term (up to 5 years). ${ }^{31}$

In the present study, the increased distance covered in the 6MWT reflects cardiopulmonary and skeletal muscle adaptations following the PT, such as increased RMS and PMS. These changes probably resulted in a greater functional capacity to perform the daily tasks, as well as an increase in the quality of life of the volunteer.

\section{Hemodynamic variables}

In this study, in addition to the improvement in distance and mean velocity demonstrated in the 6MWT, there was a reduction in SBP and DBP after the $11^{\text {th }}$ training session, associated with a decrease in Rate-pressure product at rest and during the 6MWT.

In the post-HT period, the heart remains denervated with an autonomic derangement of sympathetic and parasympathetic activity, usually for up to one year after the HT., ${ }^{4,32}$ Initially, the transplanted heart presents changes in the responses to exercise, such as chronotropic and inotropic incompetence, reduced cardiac output and systolic volume, as well as systolic and diastolic dysfunction. There is also an increase in mean pulmonary artery pressure and vascular resistance at rest and during exercise. These changes result in reduced exercise capacity after HT., ${ }^{4,26}$ In view of this, the increase in cardiac output (HO) elicited during exercise is mainly due to increased venous return via active muscular pumping, obeying the Frank-Starling law. ${ }^{4,26}$

The performance of exercises in heated water promotes an increase in venous return due to hydrostatic pressure, which increases with water depth and density, and favors the diuretic reaction, due to the suppression of the hormones, contributing to better circulation and reduction of edema. ${ }^{33}$

\section{SBP and DBP}

Regarding the blood pressure levels, physical training in heated water seems to reduce both SBP and DBP more effectively than ground-based exercises. ${ }^{27,34} \mathrm{Immersion}$ in water promotes a decrease 
in peripheral resistance due to dilation of the arterioles, with a subsequent fall in BP. With the reduction of the effects of gravity in the swimming pool, the fluid of the lower limbs will be directed toward the chest, which increases venous return and in turn, stimulates the baroreceptors to reduce the $\mathrm{BP}^{34,35}$

The reduction of both SBP and DBP (of approximately $10 \mathrm{mmHg}$ ) observed in the present study is in agreement with the findings of other authors. Castro et al. ${ }^{31}$ compared the behavior of SBP and DBP in post-transplant patients, identifying a greater reduction in blood pressure levels in the group that exercised in heated water (6.6 to $12.3 \mathrm{mmHg}$ ) in relation to the patients who performed ground-based exercise (5 to $8.3 \mathrm{mmHg}$ ) for 12 weeks. ${ }^{9}$ The main effects regarding $\mathrm{BP}$ reduction are related to a reduction in vascular resistance, which in turn is associated with lower sympathetic system activity, improved baroreflex sensitivity and endothelial function, as well as reduced arterial stiffness. ${ }^{36}$

\section{Rate-pressure product}

Properly prescribed and properly performed physical training is able to reduce resting HR and HR with submaximal workloads, as well as SBP and DBP values in patients after HT. ${ }^{34,35}$ In the present study, there was a reduction of RPP at rest and during the execution of the 6MWT after the physical training. Likewise, Marconi \& Marzorati ${ }^{36}$ observed a reduction in RPP for the same workload. ${ }^{37}$ These results reflect improvements in the functional capacity and cardiac function exemplified by the increase in the LVEF (\%).

\section{LVEF (\%)}

Left ventricular function, evaluated through the Doppler echocardiogram, consists of the filling and emptying of the left ventricle, which determines the LVEF (\%) and consequently translates the ability of the left ventricle to eject blood into the aorta during systole. ${ }^{38}$ The LVEF (\%) involves both the final systolic volume (FSV) and the final diastolic volume (FDV), with values $\geq 55 \%$ (Teicholz method) being considered normal for healthy adults. ${ }^{38}$

In cardiac transplant patients there is a reduction in systolic volume at rest and during exercise, when compared to healthy individuals. ${ }^{39}$ In these patients, cardiac function is mainly limited by chronotropic incompetence and diastolic dysfunction, which can be defined by the deficit of ventricular relaxation and increase of intraventricular pressures, with consequent elevation of pulmonary capillary pressure. These changes limit the increase in cardiac output mainly in higher intensity exercise. ${ }^{40}$

In the present case, the initial records of the LVEF (\%) show LV diastolic dysfunction, with signs of low output. Diastolic dysfunction appears to be a result of HBP and the use of immunosuppressant medications $(4,5)$. After HT, the initial ECO shows preserved systolic function with the LV of normal dimensions. The final exam shows the LV with normal dimensions, non-hypertrophied and with preserved systolic function, with the LVEF (\%) estimated at $70 \%$. In this context, the probable participation of the aquatic physical training in this improvement of cardiac function can be hypothesized. Despite the short time after the HT $(<1$ year), aquatic training may have contributed, even partially, to the reduction of the post-load and improvement of endothelial and systolic function.

Gabrielsen et al. ${ }^{40}$ demonstrated that physical training in warm water improves cardiac function through increased diastolic filling and HR reduction resulting in increased systolic volume and LVEF in patients with HF who exhibited systolic and diastolic dysfunction. ${ }^{41}$ On the other hand, Nytroen et al. ${ }^{41}$ submitted 48 post-treatment ( 8 years) patients to physical training for 12 weeks, with high intensity exercises ( 85 to $95 \%$ of maximal HR) and described a maximal $\mathrm{VO}_{2}$ increase, a reduction of resting $\mathrm{HR}$ and no change in ejection volume. ${ }^{42}$

\section{Walking speed test}

The WST is an important measure that correlates with functional capacity and dynamic balance, the potential of which is to predict functional decline as well as the risk of falls. ${ }^{43}$ The strength of the LLs and the dynamic balance are directly related to the walking speed measured in the WST. ${ }^{43}$

Bento et al. ${ }^{43}$ tested the balance of 65 women aged over 60 years, before and after a 12-week exercise protocol in water. The authors showed that the training in water had a positive effect on dynamic balance, allowing the study subjects to increase their walking speed. The most plausible hypothesis is that water turbulence offers a valuable stimulus for the musculature of the LLs, as it promotes an improvement in their ability to react when confronting a disturbance. ${ }^{44,45}$ Similarly, other studies also corroborate the increase in dynamic balance in the older adults after performing aquatic exercises that include rapid movements with change of direction. ${ }^{46,47}$ Thus, these exercises demand rapid production of torque against the water turbulence, which favors the improvement of the functional response of the dynamic balance.

The present study confirms these findings, since at baseline the volunteer performed the $10 \mathrm{~m}$ WST in $18.3 \mathrm{~s}$ with a mean speed of $0.55 \mathrm{~m} / \mathrm{s}$. After the training, there was an increase in the walking speed of $0.43 \mathrm{~m} / \mathrm{s}$, with the $10 \mathrm{~m}$ covered in $10.2 \mathrm{~s}$ and a mean speed of $0.98 \mathrm{~m} / \mathrm{s}$. According to Cruz et al. ${ }^{47}$ a walking speed of $0.8 \mathrm{~m} / \mathrm{s}$ is the cut-off point for the diagnosis of sarcopenia, according to the algorithm proposed by the European Working Group on Sarcopenia. ${ }^{48}$

\section{Quality of life}

According to Trevizan et al. ${ }^{48}$ heart transplantation per se is already a predictor of QoL improvement for transplant patients because they feel satisfied due to overcoming the pre-transplant difficulties. ${ }^{49}$ Likewise, other studies have shown that HT surgery in patients with refractory heart failure presents better results in relation to QoL, as they see an improvement in the physical condition..$^{50,51}$

The results obtained in the present study show that the exercise protocol in water increased the QoL improvement. There was improvement in all domains of the questionnaire, mainly in the functional capacity and limitations in physical aspects, with increases of $200 \%$ and $75 \%$, respectively. Regarding the social and emotional aspects, the gain was $100 \%$ and $33.34 \%$.

Improvement in functional capacity and peripheral muscle strength with greater exercise tolerance reflect the greater ability to spend more time doing activities that require physical effort or even doing leisure activities. ${ }^{17}$ Thus, there is an increase in QoL in the physical and functional fields. In relation to the $100 \%$ gain in the social aspects domain observed in the present study, this indicates that the improvement of the physical capacity and the tolerance to the exercises can contribute to greater participation in social interaction activities, such as those of leisure. ${ }^{17,52}$ Studies emphasize the importance of physical training for the improvement of QoL, since 
depression, anxiety and fatigue are conditions commonly present in post-HT subjects. ${ }^{53}$ In this way, physical training can positively impact the emotional aspect since it reduces these conditions..$^{52,53}$

\section{Limitations}

This was a case study with a single volunteer, therefore generalizations cannot be made. However, since the goal was specific physical training in a relatively rare particular case, the type of design fits perfectly. The training period should have been extended to more weeks and the follow-up should have been performed after a longer period. In addition, the description of other data, from complementary exams, would be important to establish more correlations, as well as clinical and functional implications.

\section{Conclusion}

The present study demonstrated that a specific 10-week waterbased exercise protocol, targeting a volunteer that underwent a recent HT, resulted in significant gains in muscle strength, improvement in functional capacity, walking speed and LVEF (\%), as well as improvements in the quality of life.

\section{Acknowledgements}

The authors are pleased to acknowledge the Universidade de Itaúna, who provided all the resources to do the study.

\section{Conflict of interest}

The authors declare no conflict of interest.

\section{References}

1. Ravis E, Theron A, Mancini J, et al. Severe right ventricular dysfunction is an independent predictor of pre- and post-transplant mortality among candidates for heart transplantation. Arch Cardiovasc Dis. 2017;110(3):139-148.

2. Registro Brasileiro de Transplantes de 2017. Ano XXI Num..

3. Williams TJ, McKenna MJ. Exercise limitation following transplantation. Compr Physiol. 2012;2(3):1937-79.

4. Grigioni F, Specchia S, Maietta P, et al. Changes in exercise capacity induced by heart transplantation: prognostic and therapeutic implications. Med Sci Sports. 2011;21(4):519-725.

5. Korzeniowska KI, Bilińska M, Dobraszkiewicz WB, et al. The influence of water-based training on arrhythmia in patients with stable coronary artery disease and preserved left ventricular function. Cardiol $J$. 2016;23(1):93-9.

6. Garzon M, Juneau M, Dupuy O, et al. Cardiovascular and hemodynamic responses on dryland vs. immersed cycling. J Sci Med Sport. 2015;18(5):619-23.

7. Guimaraes GV, De Barros Cruz LG, Fernandes SMM, et al. Heated water-based exercise training reduces 24-hour ambulatory blood pressure levels in resistant hypertensive patients: A randomized controlled trial (HEx trial). Int J Cardiol. 2014;172(2):434-41.

8. Castro RE, Guimarães GV, Da Silva JMR, et al. Postexercise hypotension after heart transplant: Water-versus land-based exercise. Med Sci Sports Exerc. 2016;48(5):804-10.

9. Adriana J, Souza MA De, De RAB, et al. Efeitos de um programa de exercícios em um adulto com acidente vascular encefálico após transplante cardíaco. Insuficiencia cardiac. 2017;12(1):1-10.
10. Crapo RO, Casaburi R, Coates AL, et al. ATS statement: Guidelines for the six-minute walk test. Am J Respir Crit Care Med. 2002;166(1):111-7.

11. Martinez BP, Batista AKMS, Ramos IR, et al. Viability of gait speed test in hospitalized elderly patients. J Bras Pneumol. 2016; 42(3):196-202.

12. Neder JA, Andreoni S, Lerario MC, et al. Reference values for lung function tests. II. Maximal respiratory pressures and voluntary ventilation. Brazilian J Med Biol Res. 1999;32(6):719-27.

13. Ciconelli RM, Ferraz MB, Santos W, et al. Tradução para a língua portuguesa e validação do questionário genérico de avaliação de qualidade de vida SF-36 (Brasil SF-36). Revista Brasileira De Reumatologia. 1999;39(3):143-50.

14. Shephard RJ, Kavanagh T, Mertens DJ, et al. The place of perceived exertion ratings in exercise prescription for cardiac transplant patients before and after training. Br J Sports Med. 1996;30:116-121.

15. Campos FO, Zielinsky P, Ortiz J, et al. Guideline for indication and utilization of echocardiography in clinical practice. Arq Bras Cardiol. 2004;82(supp12):11-34.

16. Dall CH, Gustafsson F, Christensen SB, et al. Effect of moderate- versus high-intensity exercise on vascular function, biomarkers and quality of life in heart transplant recipients: A randomized, crossover trial. $J$ Hear Lung Transplant. 2015;34(8):1033-41.

17. Lavie CJ, Arena R, Earnest CP. High-intensity interval training in patients with cardiovascular diseases and heart transplantation. $J$ Heart and Lung Transplant. 2013;32(11):1056-1058.

18. Branson RD. The Scientific Basis for Postoperative Respiratory Care. Respiratory Care. 2013;58(11):1974-1984.

19. Stephens RS, Whitman GJ. Postoperative critical care of the adult cardiac surgical patient. Crit Care Med. 2015;43(7):1477-97.

20. Kinugasa Y, Yamamoto K. The challenge of frailty and sarcopenia in heart failure with preserved ejection fraction. Heart. 2017; 103(3):184-189.

21. Yamada K, Kinugasa Y, Sota T, et al. Inspiratory Muscle Weakness is Associated With Exercise Intolerance in Patients With Heart Failure With Preserved Ejection Fraction: A Preliminary Study. J Card Fail. 2016;22(1):38-47.

22. Montemezzo D, Fregonezi GA, Pereira DA, et al. Influence of inspiratory muscle weakness on inspiratory muscle training responses in chronic heart failure patients: A systematic review and meta-analysis. Arch Phys Med Rehabil. 2014;95(7):1398-407.

23. Forestieri P, Guizilini S, Peres M, et al. A Cycle ergometer exercise program improves exercise capacity and inspiratory muscle function in hospitalized patients awaiting heart transplantation: a pilot study. Braz $J$ Cardiovasc Surg. 2016;31(5):389-395.

24. Cahalin LP, Arena R, Guazzi M, et al. Inspiratory muscle training in heart disease and heart failure: a review of the literature with a focus on method of training and outcomes. Expert Rev Cardiovasc Ther. 2013;11(2):161177.

25. Carvalho VO, Barni C, Teixeira- IS, et al. Exercise capacity in early and late adult heart transplant recipients. Cardiol J. 2013;20(2):178-83.

26. Bento PCB, Pereira G, Ugrinowitsch C, et al. The effects of a water-based exercise program on strength and functionality of older adults. $J$ Aging Phys Act. 2012;20(4):469-83.

27. Cuoco A, Callahan DM, Sayers S, et al. Impact of muscle power and force on gait speed in disabled older men and women. J Gerontol A Biol Sci Med Sci. 2004;59(11):1200-6.

28. Chen SY, Lu PC, Lan C, et al. Six-minute walk test among heart transplant recipients. Transplant Proc. 2014;46(3):929-33. 
29. Wegrzynowska TK, Rudzinska E, Lazorczyk M, et al. Distance covered during a six-minute walk test predicts long-term cardiovascular mortality and hospitalisation rates in men with systolic heart failure: an observational study. Journal of Physiotherapy. 2013;59(3):177-187.

30. Castel MA, Mendez F, Tamborero D, et al. Six-minute walking test predicts long-term cardiac death in patients who received cardiac resynchronization therapy. Europace. 2009;11(3):338-342.

31. Ciolac EG, Castro RE, Greve JM, et al. Prescribing and regulating exercise with RPE after heart transplant: a pilot study. Med Sci Sports Exerc. 2015;47(7):1321-7.

32. Tei C, Horikiri Y, Park JC, et al. Acute hemodynamic improvement by thermal vasodilation in congestive heart failure. Circulation [online]. 1995;91(10):2582-90.

33. Guimarães GV, Cruz LGB, Tavares AC, et al. Effects of short-term heated water-based exercise training on systemic blood pressure in patients with resistant hypertension. Blood Press Monit. 2013;18(6):342-5.

34. Bocalini DS, Bergamin M, EvangelistaAL, et al. Post-exercise hypotension and heart rate variability response after water- and landergometry exercise in hypertensive patients. Plos one. 2017;12(6):e0180216.

35. Carpio RE, Moncada JJ, Salazar-RW, et al. Acute Effects of Exercise on Blood Pressure: A Meta-Analytic Investigation. Arq Bras Cardiol. 2016;106(5):422-433.

36. Marconi C, Marzorati M. Exercise after heart transplantation. Eur J Appl Physiol. 2003;90(3-4):250-9.

37. Badano LP, Miglioranza MH, Edvardsen T, et al. European Association of Cardiovascular Imaging/Cardiovascular Imaging Department of the Brazilian Society of Cardiology recommendations for the use of cardiac imaging to assess and follow patients after heart transplantation. Eur Heart J Cardiovasc Imaging. 2015;16(9):919-48.

38. Salles AF, Machado CV, Cordovil A, et al. A Elevação da Pressão Arterial Sistólica Durante o Teste Ergométrico Após Transplante Cardíaco: Correlação com o Quadro Clínico e a Função Ventricular Avaliada pela Ecocardiografia Sob Estresse com Dobutamina. Arq Bras Cardiol. 2006;87(5):628-633.

39. Marconi C, Marzorati M. Exercise after heart transplantation. Eur J Appl Physiol. 2003;90(3-4):250-9.

40. Gabrielsen A, Sorensen VB, Pump B, et al. Cardiovascular and neuroendocrine responses to water immersion in compensated heart failure. Am J Physiol Heart Circ Physiol . 2000;279(4):H1931-40.
41. Nytroen K, Rustad LA, Aukrust P, et al. High-Intensity Interval Training Improves Peak Oxygen Uptake and Muscular Exercise Capacity in Heart Transplant Recipients. Am J Transplant. 2012;12(11):3134-42.

42. Bootsman NJM, Skinner TL, Lal R, et al. The relationship between physical activity, and physical performance and psycho-cognitive functioning in older adults living in residential aged care facilities. $J \mathrm{Sci}$ Med Sport. 2017;21(2):173-178.

43. Bento PCB, Lopes MFA, Cebolla EC, et al. Effects of Water-Based Training on Static and Dynamic Balance of Older Women. Rejuvenation Res. 2015;18(4):326-31.

44. Padua E, Campoli F, Manzi V, et al. Water versus land-based exercises as physical training programs in elderly. J Sports Med Phys Fitness. 2017.

45. Bergamin M, Ermolao A, Tolomio S, et al. Water- versus land-based exercise in elderly subjects: effects on physical performance and body composition. Clin Interv Aging. 2013;8:1109-17.

46. Oh S, Lim J-M, Kim Y, et al. Comparison of the effects of water- and landbased exercises on the physical function and quality of life in communitydwelling elderly people with history of falling: A single-blind, randomized controlled trial. Arch Gerontol Geriatr. 2015;60(2):288-93.

47. Cruz JAJ, Baeyens JP, Bauer JM, et al. Sarcopenia: European consensus on definition and diagnosis. Age Ageing. 2010; 39(4):412-23.

48. Trevizan FB, Miyazaki MCOS, Silva YLW, et al. Quality of Life, Depression, Anxiety and Coping Strategies after Heart Transplantation. Braz J Cardiovasc Surg. 2017;32(3):162-170.

49. Pfeifer PM, Ruschel PP, Bordignon S. Coping strategies after heart transplantation: psychological implications. Rev Bras Cir Cardiovasc. 2013;28(1):61-8.

50. Czyzewski L, Torba K, Jasinska M, et al. Comparative analysis of the quality of life for patients prior to and after heart transplantation. Ann Transplant. 2014;19:288-94.

51. Anderson L, Dall CH, Nguyen TT, et al. Exercise-based cardiac rehabilitation in heart transplant recipients (Protocol). Cochrane Database of Systematic Reviews. 2016(6).

52. Aguiar MIF de, Farias DR, Pinheiro ML, et al. Qualidade de vida de pacientes submetidos ao transplante cardíaco: aplicação da escala Whoqol-Bref. Arq Bras Cardiol. 2011;96(1):60-8. 